\title{
Model NbTi Helical Solenoid Fabrication and Test Results
}

\author{
N. Andreev, E. Barzi, G. Chlachidze, D. Evbota, V. S. Kashikhin, V. V. Kashikhin, M. J. Lamm, A. \\ Makarov, I. Novitski, D. F. Orris, M. A. Tartaglia, J. C. Tompkins, D. Turrioni, D. Walbridge, M. Yu, \\ A. V. Zlobin
}

\begin{abstract}
A program to develop model magnets for a helical cooling channel is under way at Fermilab. In the first steps of a planned sequence of magnets, two four-coil helical solenoid models with $300 \mathrm{~mm}$ aperture have been fabricated and tested. These two models, HSM01 and HSM02, used insulated NbTi Rutherford cable wound onto stainless steel rings with spliceless transitions between coils. Strip heaters were included for quench protection of each coil, and the coils were epoxy-impregnated after winding inside the support structures. Based on the results of the first model the second model was made using a cable with optimized cross-section, improved winding and epoxyimpregnation procedures, enhanced ground insulation, and included heat exchange tubing for a test of conduction cooling. We report on the results and lessons learned from fabrication and tests of these two models.
\end{abstract}

Index Terms-Helical Cooling Channel, Helical Solenoid, NbTi, Superconducting Magnet

\section{INTRODUCTION}

$\mathrm{T}$ HE particle physics energy frontier future presents tremendous technological challenges to the accelerator community. Along one possible path to the future, a major international effort is under way to advance the technologies required to collect, cool, and accelerate intense muon beams for a future neutrino factory or muon collider. It is now well recognized that a number of very challenging superconducting magnets must be developed to meet specific requirements throughout such a machine [1].

The function of muon cooling is critical to success of a muon collider approach, but the concept is purely theoretical at present. A major undertaking to demonstrate this is moving forward in the Muon Ionization Cooling Experiment (MICE) [2]. Independent of this, possible cooling channel designs based on the ionization cooling concept have been developed. One design concept in particular has resulted from a collaboration, supported by the U.S. Department of Energy through a number of SBIR/STTR (Small Business Innovative Research) grants, of Muons, Inc. with Fermilab: the utilization of a Helical Cooling Channel (HCC). The HCC would rely

Manuscript received 12 September 2011. This work was supported in part by Fermi Research Alliance, LLC, under contract No. DE-AC02-07CH11359 with the U.S. Department of Energy.

All authors are with the Fermi National Accelerator Laboratory, Batavia, IL 60510 USA (corresponding author phone: 630-840-3890; fax: 630-8408079; e-mail: tartaglia@fnal.gov). upon fixed-period helical solenoid magnets to provide the required solenoid, helical dipole, and helical gradient field components needed to constrain the muons to move along the helical trajectory; 6D cooling would occur as muons traverse and ionize a suitable medium within the magnet bore, and longitudinal momentum is restored by periodic RF acceleration.

A particular HCC has been simulated where cooling would be done in four stages, and a set of required magnet parameters was specified [3]. Two magnet designs were conceived to provide the desired fields [4], and a 6D muon cooling experiment, MANX, was proposed to demonstrate the use of the HCC [5]. One option is to use a large solenoid with external helical dipole and quadrupoles; the second option is to use a sequence of short circular coil rings whose centers are placed on a helical beam trajectory. As discussed in [6] this second novel design has the advantages of requiring half the coil and superconductor volume, has much lower peak field, reduced stored energy and coil stresses, naturally produces the dipole and quadrupole fields, and can be built.

In this HCC design [3] the magnet strength progressively increases, while the magnet aperture decreases, from stage to stage. The field strength required in the first two stages requires $\mathrm{Nb} 3 \mathrm{Sn}$ superconductor, while the later stages may need high temperature superconductor. Motivated by these studies, Fermilab formed plans to develop and test a progression of model magnets of the helical solenoid (HS) design. We report in this paper on the first two models in that progression, which have been built from $\mathrm{NbTi}$ conductor.

\section{Helical SOlENOID DESIGN}

The first model magnets were intended to develop practical experience with the construction and performance of superconducting helical solenoids. The size of the model magnets was limited by the $640 \mathrm{~mm}$ inner diameter of the test dewar at the Fermilab Vertical Magnet Test Facility (VMTF). A short four-coil design [7] was deemed sufficient to explore all of the fabrication complexities and structural stresses of a long HS, while allowing fairly large aperture rings and generating a peak field of order $5 \mathrm{~T}$.

The four coils are built up from a stainless steel end flange, successively wound in the "hard way bend" direction using pre-insulated NbTi SSC dipole inner cable (30 strand) around an insulated stainless steel (S.S.) ring, then surrounded by an outer S.S. ring. Each coil ring is aligned with pins and skip- 
welded in place before winding the next coil. The helical structure allows all four coils to be continuously wound with a smooth transition between layers and no splices. Fig. 1 shows the schematic layout of the short HS model, and Fig. 2 shows a photograph of the HS during winding. After winding is compete, a top end flange is welded in place, and the assembly is vacuum impregnated with epoxy. A S.S. outer skin is welded around the outer diameter of the end flanges to react against Lorentz forces that tend to straighten the helix.

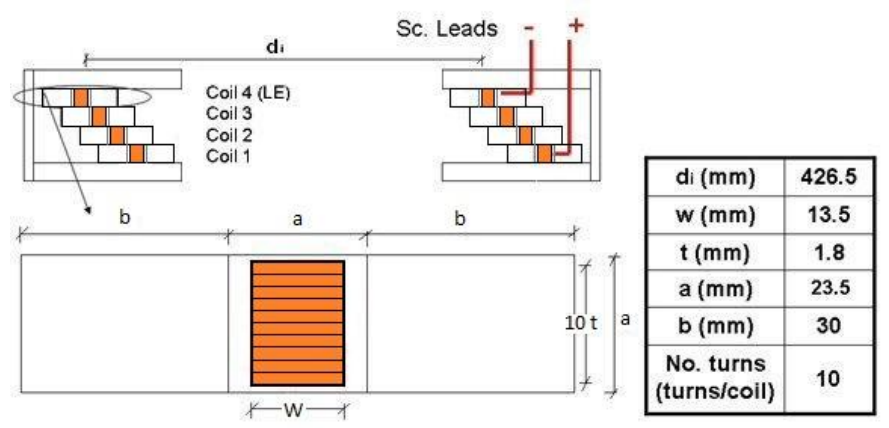

Fig. 1. Schematic cutaway side view of HSM02 helical four coil structure with detail view of coil ring cross section and dimensions.

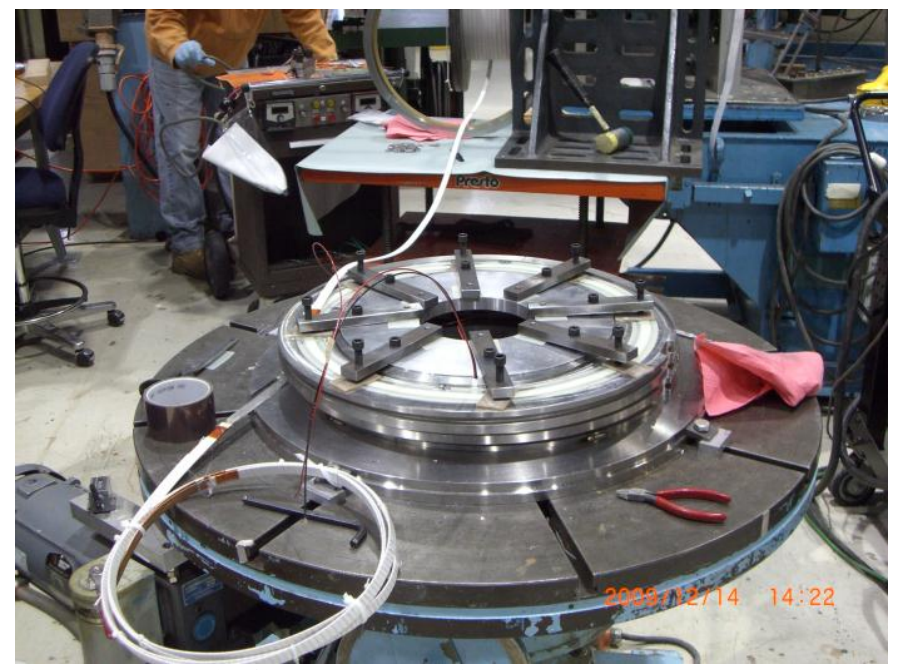

Fig. 2. Photograph of HSM02 during winding.

\section{FABRICATION AND TEST HISTORY}

A great deal was learned from fabrication and testing of the first model, HSM01 [8]. The use of keystoned cable made it difficult to wind the coils with the desired packing factor; only 37 of the planned 40 total turns were achieved. A high resistance electrical short developed between the coil and S.S. structure, which limited the energy extraction resistance to prevent high voltage development at quench. During the quench testing, motion of the superconducting leads in the large solenoid fringe field resulted in a lead quench, and fault to ground which fortunately did not result in damage or end the test. The magnet reached a plateau quench current (12 kA) well above the nominal operating point $(9.6 \mathrm{kA})$. Also, the strain gauge measurements were successful in confirming the finite element mechanical model predictions [7], which indicated that stresses in the structure are well within the maximum allowable, even well above the nominal operating point to the expected maximum quench current. Relating the magnetic field measurements to model predictions clarified the need for better coordinate system determination by well defined survey and alignment features.

After completing the HSM01 test the model was cut into several sections, ends were polished and inspected. Major findings include: epoxy voids near S.S. ring interfaces and thick regions of epoxy around coils that may crack, bending of G10 insulation, some deformed NbTi strands, and one section of one cable layer with a high resistance short to ground.

While essentially the same design, HSM02 was improved in several ways [9]. First, in order to ensure a fit of 10 turns per layer, the ring height was increased from 20 to $23.5 \mathrm{~mm}$. Also the SSC cable was annealed and re-sized to a rectangular cross section; subsequent short sample measurements of extracted strands showed no change in the strand critical current. G10 insulation layers were increased in width between layers to prevent shorts in the transition regions, and in thickness between coil and S.S. rings to reduce epoxy. A second impregnation step was added, with a mica powder mixed in to displace the plain epoxy in large void regions and prevent cracking. Design of the support for superconducting leads was improved, and precise alignment features were machined in the non-lead end flange.

These improvements led to improved results in HSM02: each coil layer with 10 turns, no insulation problems between coils or ground, no superconducting lead quenches. In addition, strip heaters for quench protection, which had been on the outer perimeter of coils in HSM01, were moved to the coil inner radius in HSM02 with expectation of improving thermal contact with the coils. Both magnets were tested in a boiling liquid helium bath, however interest in the possibility of cooling the coils indirectly led to the addition in HSM02 of copper tubing attached to the outside perimeter around each of the four coils, and an attempt was made to study the effectiveness of conduction rather than helium bath cooling.

\section{TEST RESUltS}

\section{A. Magnetic Measurements}

Magnetic measurements on HSM01 and HSM02 were made during cold testing using a $10 \mathrm{~T}$ 3D Senis Hall probe in a warm bore tube centered on the aperture. Peak axial field and profile for both magnets agreed well with Opera3D predictions and is linear up to $5 \mathrm{kA}$ (there is no magnetic material). HSM01 full aperture was mapped at 10 A by an array of three 1D Group3 probes at room temperature, with axial scans at a radius of 4 inches at 45 degree angle steps. A comparison is shown in Fig. 3 of several representative measurements with the corresponding model prediction of axial field shapes. A similar comparison for one transverse component $\left(B_{x}\right)$ is shown in Fig.4. The field profiles are in reasonably good agreement, but some discrepancies between model and data remain due to the (motion, probe, and model) coordinate system definitions and offsets.

Warm measurements of HSM02 using a new improved measurement system, and with closer attention to coordinate systems and survey, are pending. 


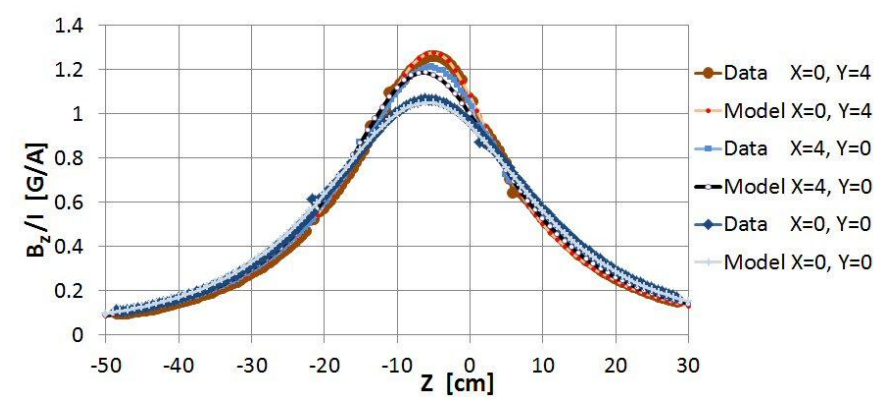

Fig. 3. Comparison of HSM01 axial field magnetic model and measurement.

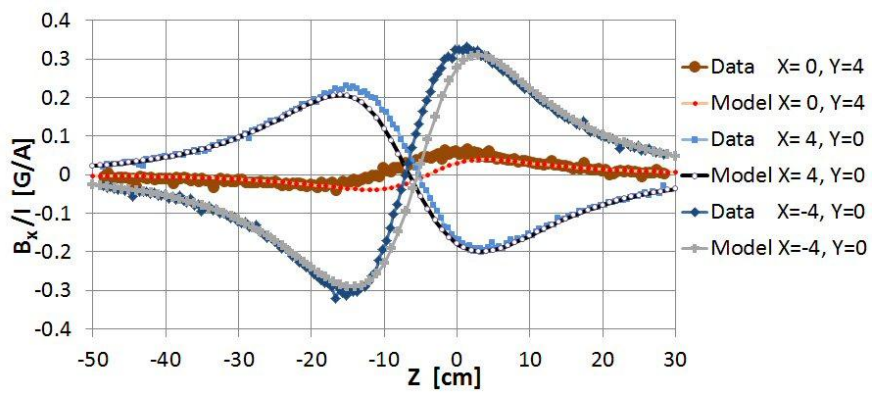

Fig. 4. Comparison of HSM01 transverse $\left(B_{x}\right)$ field magnetic model and measurement.

\section{B. Quench Performance}

The good agreement between magnetic model and measurements lends confidence to the calculated peak coil fields. The predicted maximum quench current for HSM02 is $15 \mathrm{kA}$, shown in Fig. 5, and 16.5 kA for HSM01 with fewer coil turns. The peak field is slightly higher on the two end coils. Fig. 6 shows the quench histories in the first cold test cycle for both magnets. They are very similar in terms of the rate of quench training, occasional erratic (reduced) quench current, and lack of improvement with lowered temperature indicative of mechanical imperfection. Eventually HSM02 reached the expected maximum; while HSM01 training was ended at $85 \%$, the HSM02 trend suggests HSM01 may well have reached the maximum if training had been continued.

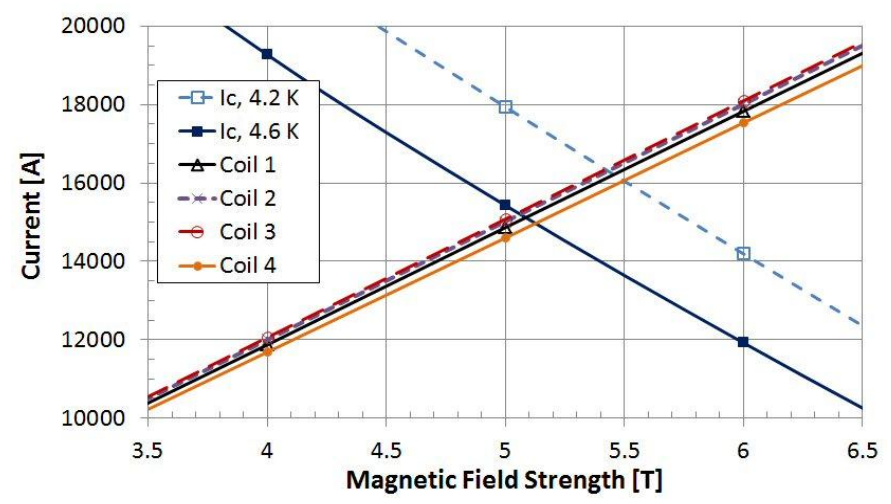

Fig. 5. Quench current prediction for HSM02, showing load lines and SSC cable short sample measurements at $4.2 \mathrm{~K}$, and parameterized $4.6 \mathrm{~K}$ curve.

Although most quenches were conducted at a $50 \mathrm{~A} / \mathrm{s}$ current ramp rate, other ramp rates were explored in both model magnets. No significant ramp rate dependence was seen in the quench current, up to $500 \mathrm{~A} / \mathrm{s}$.

HSM02 was warmed to room temperature and tested again at $4.6 \mathrm{~K}$, to study re-training after a thermal cycle (TC). Fig. 7 shows the overlaid quench training histories of the first and second TC, showing that the retraining is quite short.

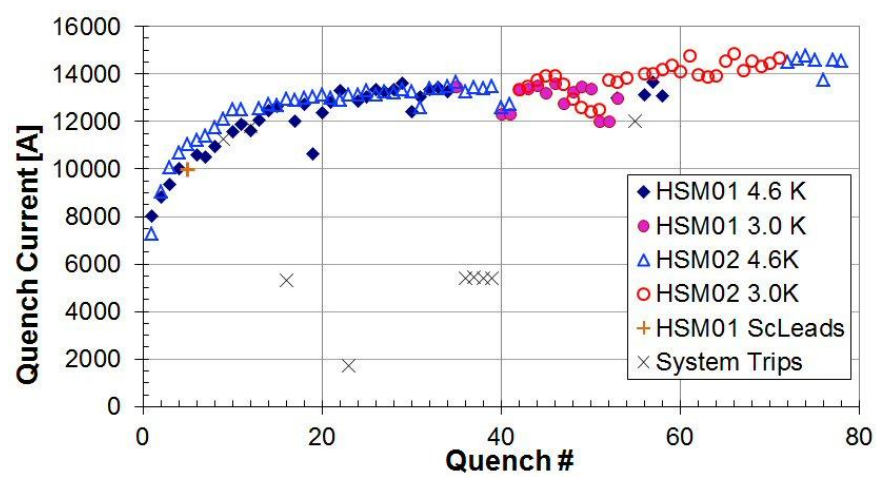

Fig. 6. Overlay of HSM01 and HSM02 first thermal cycle quench histories. System trip events resulted from power supply regulation and test stand current lead issues.

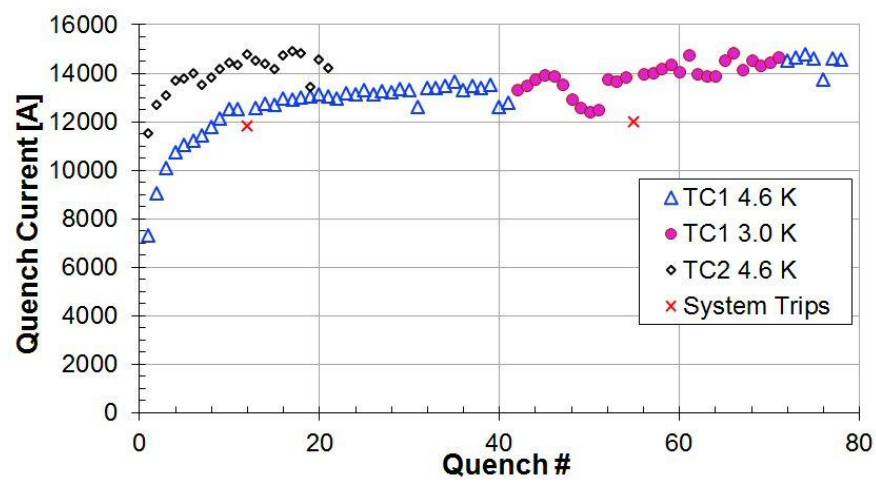

Fig. 7. Overlay of HSM02 quench histories in first and second thermal cycle.

Coils were instrumented with one voltage tap at each transition layer and at the leads, so quench locations were determined only at the coil level. Fig. 8 shows the frequency distribution of quenches for both model magnets. Clearly the end coils experience the majority of quenches, in both magnets and after a thermal cycle. In all tests, only two HSM02 quenches started in two coils (nearly simultaneously), and these both involved one end coil and its adjacent coil. Quench voltage development times were $\sim 5-10 \mathrm{~ms}$ (faster at higher current), which for typical quench propagation velocity $(\sim 20 \mathrm{~mm} / \mathrm{ms})$, suggests that most quench origins are unlikely to be in the transition region between coil rings (they would have propagated into both coils in the available time).
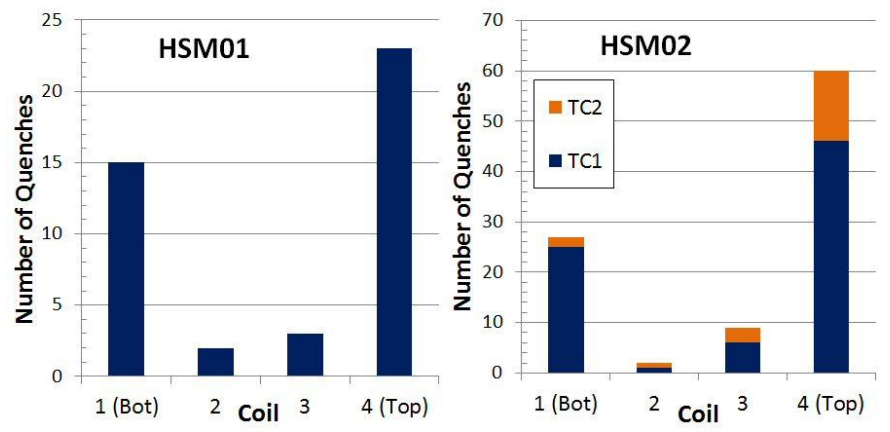

Fig. 8. Frequency distribution of HSM01 and HSM02 quench locations.

\section{Protection Heater Study}

Strip heater effectiveness tests were made in the HSM02 second thermal cycle, to study performance for future use in 
long HS quench protection. Each coil heater was studied at $4.6 \mathrm{~K}$ as a function of the magnet current and discharge heater power supply (HPS) voltage, all at fixed capacitance $(4.8 \mathrm{mF})$. Fig. 9 shows the quench development time, from heater discharge to quench detection, for each coil as a function of HPS voltage and current settings. Some small variation between coils is seen, but they are consistent in general, and reasonable values for heater settings are easily achieved.

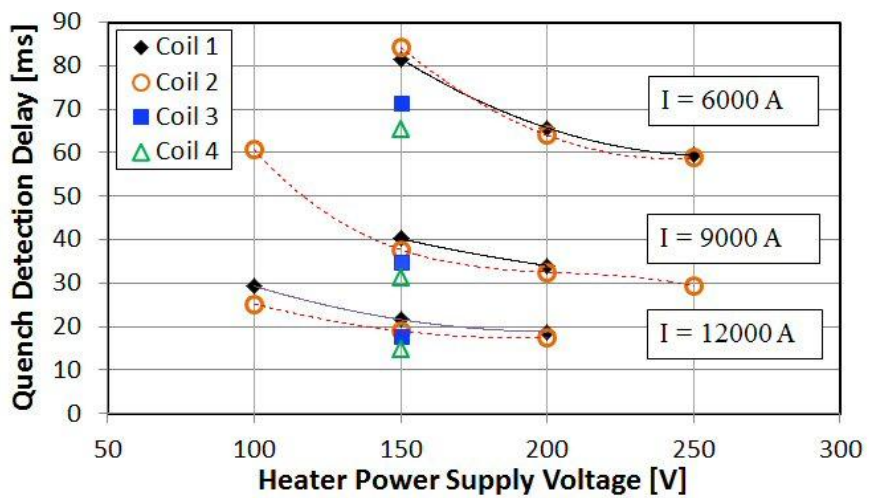

Fig. 9. HSM02 quench delay versus protection heater voltage at three magnet current settings.

\section{RRR measurement}

The electrical properties of the copper stabilizer in the coils are relevant to quench protection, and are measured as a standard part of the superconducting magnet test program. The residual resistivity ratio (RRR) of the conductor is estimated as the ratio of room temperature resistance to that just after the superconductor transition at about $9 \mathrm{~K}$, as the magnet warms up slowly following the cold test. Table 1 shows these RRR measured values for the coils in HSM01 and HSM02. Lower gain amplifiers were used in HSM01 measurements than for HSM02 (and one readout channel failed), so cold voltage measurements limited the RRR value precision. Nevertheless, the likely reason for RRR reduction in HSM02 is reworking of the cable to remove the keystone angle.

TABLE 1 RRR MEASUREMENTS

\begin{tabular}{ccc}
\hline \hline Coil & HSM01 & HSM02 \\
\hline 4 (Top) & -- & 102.4 \\
3 & 142.2 & 101.7 \\
2 & 140.5 & 101.0 \\
1 (Bottom) & 155.7 & 102.7 \\
\hline \hline
\end{tabular}

\section{E. Conduction Cooling Study}

As described earlier HSM02 outer S.S. coil rings were surrounded with copper tubing, which were wound continuously from the bottom coil to the top coil. Following the cold quench performance tests, an attempt to measure the conduction cooling behavior of this configuration was made. The VMTF lacks an independent helium supply for connection to this tubing, so the test was made using liquid nitrogen (LN2) instead. Also the VMTF dewar is not designed to be a vacuum vessel, but a vacuum of 0.12 Torr was achieved before introducing LN2 to the tubing, (bottom coil first), and the vacuum slowly improved to 0.03 Torr, still two orders of magnitude higher than good insulating vacuum.

Temperature sensors on each coil and both end flanges were monitored as a function of time until all coils reached the LN2 temperature, which (at 46 PSIG) was $92 \mathrm{~K}$, about 24 hours later. Although the coil temperatures responded in the right order (bottom to top), not much can be concluded from this test because the temperature sensors lack calibration in the range between 300 and $80 \mathrm{~K}$ (they are dense at lower temperatures). Thus the individual coil sensor temperatures varied by over $20 \mathrm{~K}$ when they reached $92 \mathrm{~K}$.

Nevertheless, upon removing the magnet assembly from the test dewar, an important lesson about the cooling tubing design was learned. The neatly arranged copper tubing and stainless steel connections to the LN2 supply were found to have been distorted and had signs of strong electrical arcing. The obvious conclusion is that the continuous copper loop created an efficient 10:1 transformer, generating Lorentz forces in the unconstrained supply tubing and arcs at the contact points.

\section{CONCLUSION}

Two short 4-coil helical solenoid model magnets were built and tested, to develop the techniques for fabricating longer HS magnets that could be used in a Helical Cooling Channel for a muon accelerator. After testing HSM01, several design changes were made which improved performance in the second model, HSM02. Quench training of the epoxyimpregnated coils was slow, but the rate of training was nearly the same for both and they achieved quench plateau currents at $85 \%$ and $100 \%$ of the expected maximum. For both, most quenches occurred in the end coils, so one may anticipate that longer HS magnet training rate will not scale quickly with the number of coils. Ramp rate dependence is negligible, and retraining after a thermal cycle is fast. Magnetic field measurements agree well with model predictions, and efforts continue on improving coordinate systems to relate these. Although the second model had provisions for conduction cooling study, a proper facility to perform the test does not yet exist. However, the attempted study provided information to improve cooling loop design and future measurements.

\section{REFERENCES}

[1] M. Zisman, "The Muon Collider," http://www.osti.gov/energycitations/product.biblio.jsp?osti_id=1004168 [2] http://mice.iit.edu/

[3] S. A. Kahn, et al, "Magnet System for Helical Muon Cooling Channels", MOPAN117, Proceedings of PAC'07.

[4] V. S. Kashikhin, et al, "Superconducting Magnet System for Muon Beam Cooling," IEEE Trans. Appl. Supercon., Vol. 17, No. 2, pp. 10551058, June 2007.

[5] K. Yonehara, et al., "The MANX Muon Cooling Demonstration Experiment", THPMN110, Proceedings of PAC'07.

[6] V. S. Kashikhin, et al, "Magnets for the MANX 6D Muon Cooling Demonstration Experiment," MOPAS012, Proceedings of PAC'07.

[7] V. S. Kashikhin, "Four-coil superconducting helical solenoid model for muon beam cooling," WEPD013, Proceedings of EPAC'08.

[8] M. Tartaglia, et al., "Test of four coil helical solenoid magnet HSM01," FNAL, TD-09-011, April 2009.

[9] N. Andreev, et al., "HSM02 Magnet Fabrication and Test Summary," FNAL, TD-09-013, Sept. 2011. 